\title{
Clinicopathological and molecular analysis of endometrial carcinoma associated with tamoxifen
}

\author{
Julia Turbiner ${ }^{1}$, Gema Moreno-Bueno², Sonika Dahiya ${ }^{1}$, Carolina Sánchez-Estevez ${ }^{3}$, \\ David Hardisson ${ }^{4}$, Jaime Prat ${ }^{5}$, Esther Oliva ${ }^{1}$ and José Palacios ${ }^{6}$
}

${ }^{1}$ Department of Pathology, Massachusetts General Hospital, Boston, MA, USA; ${ }^{2}$ Instituto de Investigaciones Biomédicas Alberto Sols, Madrid, Spain; ${ }^{3}$ Centro Nacional de Investigaciones Oncologicas, Madrid, Spain; ${ }^{4}$ Hospital Universitario La Paz, Madrid, Spain; ${ }^{5}$ Hospital de la Santa Creu i Sant Pau, Barcelona, Spain and ${ }^{6}$ Hospital Universitario Virgen del Rocío, Sevilla, Spain

\begin{abstract}
Use of tamoxifen for treatment and prevention of breast cancer is becoming increasingly common. Tamoxifen has been associated with increased risk of endometrial carcinoma, although the exact mechanism of action is unknown. The aim of our study was to seek a possible correlation between endometrial carcinoma, tamoxifen exposure and MSI, PTEN, $\beta$-catenin and K-ras abnormalities. A group of 18 patients with endometrial carcinoma following treatment with tamoxifen were selected. A control group included 15 patients with endometrial carcinoma and associated ovarian hyperthecosis and one patient with endometrial carcinoma and adult granulosa cell tumor of the ovary, chosen because both conditions are associated with increased production of estrogen and increased risk of endometrial carcinoma development. The second control group included 27 randomly selected consecutive patients with endometrial carcinoma without identifiable associated conditions. Immunostaining for $\beta$-catenin was performed on all cases; DNA was extracted and amplified by PCR with primers for $\beta$-catenin, $K$-ras and PTEN genes. BAT-25 and BAT-26 were analyzed to assess for MSI. There were 16 endometrioid endometrial carcinomas, one mixed carcinoma and one clear cell carcinoma among patients in the tamoxifen group. All patients with ovarian hyperthecosis and adult granulosa cell tumor had endometrioid endometrial carcinoma. In the random control group, there were $\mathbf{2 6}$ endometrioid endometrial carcinomas and one carcinosarcoma. Immunohistochemical and mutational analysis for $\beta$-catenin showed abnormalities in $4 / 11$ $(36 \%)$ and $3 / 10(30 \%)$ informative cases in the tamoxifen group; $7 / 16(44 \%)$ and 4/15 (27\%) informative cases, respectively in the ovarian hyperthecosis group and $1 / 27$ random control cases $(4 \%)(P<0.05)$. Patients with tamoxifen exposure had more $K$-ras mutations and fewer PTEN mutations and MSI as opposed to controls, but the results were not statistically significant. In conclusion, there was a direct relationship between tamoxifen exposure and overexpression of $\beta$-catenin oncoprotein, which is known to play a major role in the pathogenesis of estrogen-driven, type I endometrial adenocarcinoma.
\end{abstract}

Modern Pathology (2008) 21, 925-936; doi:10.1038/modpathol.2008.49; published online 23 May 2008

Keywords: endometrial carcinoma; pathogenesis; estrogen; tamoxifen; $\beta$-catenin

Endometrial carcinoma is the most common malignancy of the female genital tract in the United States. It has been traditionally classified into two categories: type I tumors, estrogen driven and characterized by endometrioid morphology (70-80\%) and type II tumors, which are unrelated to estrogen and show serous/clear cell differentiation. ${ }^{1-3}$ Recently, the

Correspondence: Dr J Turbiner, MD, Department of Pathology (Warren 2), Massachusetts General Hospital, 55 Fruit Street, Boston, MA 02114, USA.

E-mail: jturbiner@partners.org

Received 26 December 2007; revised 06 February 2008; accepted 10 February 2008; published online 23 May 2008 molecular mechanisms underlying both types of endometrial carcinoma have been elucidated. ${ }^{2,3}$ One of the earliest and most common genetic alterations found in type I endometrial carcinoma is inactivation of PTEN, a tumor suppressor gene located on chromosome 10q23.3. Mutations or LOH in PTEN gene occur in $35-50 \%$ of type I endometrial carcino$\mathrm{ma},{ }^{2,4}$ and in up to $83 \%$ in one series. ${ }^{5} \beta$-catenin, encoded by the CTNNB1 gene, is involved in cell to cell adhesion, forming complexes with e-cadherin to maintain tissue architecture and polarity. When mutated, it acts as an oncoprotein with transcription activity downstream the Wnt signal pathway. Activating $\beta$-catenin mutations are present in about $20 \%$ 
of type I endometrial carcinoma, ranging from 14 to $44 \%{ }^{1,6-9} \mathrm{~K}$-ras encodes a protein (P21) located on the inner plasma cell membranes. It has GTPase activity and is involved in cell receptor signal transduction pathways. The constitutively activating mutations of $K$-ras protooncogene occur most frequently in codons 12 and 13 of exon 1 and have been detected in $15-30 \%$ of type I endometrial carcinoma. ${ }^{2,3}$ It seems that K-ras mutations may correlate with tumor progression and represent an early neoplastic event in endometrial carcinoma. ${ }^{10-12}$ Finally, microsatellites, short repeat DNA sequences prone to mutations during DNA replication, serve as markers of adequate function of the DNA mismatch repair family of genes. Microsatellite instability (MSI) occurs when these genes are mutated; it is found in $20-40 \%$ of type I endometrial carcinoma ${ }^{2,4,13}$ and frequently coexists with PTEN mutations. ${ }^{4}$

Estrogen stimulation is a known risk factor for the development of endometrioid endometrial carcinoma. Several mechanisms of carcinogenesis due to estrogen effect on the endometrium have been proposed. Unopposed exposure to endogenous or exogenous estrogens results in direct cell damage with increased mitotic activity, a higher likelihood of DNA errors and somatic mutations. ${ }^{14,15}$ In addition, estrogen increases the transcriptional activity of the endometrium through a complex downstream interaction of transcription factors that may likely include alteration of tumor suppressor function. ${ }^{16}$ Thus, it is known that estrogen physiologically modulates expression of PTEN in human endometrial cells. ${ }^{17-19}$

Tamoxifen, a selective estrogen receptor modulator, was first introduced in the $1970 \mathrm{~s}$ as an antiestrogenic drug for the treatment of advanced breast cancer. It is currently the most prescribed antineoplastic drug worldwide and is being used as adjuvant treatment for breast cancer, in metastatic breast cancer and, increasingly, as a means of chemoprevention in high-risk healthy women..$^{20,21}$ However, it was noticed and subsequently confirmed in large trials that tamoxifen therapy increases proliferation of endometrial cells resulting in increased prevalence of endometrial polyps, hyperplasia and carcinoma. ${ }^{22-25}$ The exact mechanism of endometrial carcinogenesis by tamoxifen is unknown. Historically, it was believed that tamoxifen acted as a partial agonist of estrogen receptors. However, recent studies have demonstrated that genes targeted by tamoxifen are largely different from those targeted by estrogen. ${ }^{26}$ Biochemical and animal experiments, as well as genetic models strongly favor an estrogen-dependent pathway with a differential gene regulation. ${ }^{27,28}$ Another hypothesis stipulates a possible genotoxic effect of tamoxifen with formation of DNA adducts, but is still controversial. ${ }^{29-33}$ The aim of our study was to analyze the expression of selected molecular markers in patients who developed endometrial carcinoma following tamoxifen exposure.

\section{Materials and methods}

\section{Samples}

Eighteen patients with endometrial carcinoma and history of tamoxifen use for breast cancer were identified from the files of the Pathology departments at the Massachusetts General Hospital, Boston, US and Hospital Universitario la Paz, Madrid, Spain. In addition, two control groups were assembled. One group consisted of 15 patients with endometrial carcinoma and associated ovarian hyperthecosis and one patient with a synchronous endometrial carcinoma and adult granulosa cell tumor of the ovary. These cases were grouped together since they converge in a common pathway of presumed increased ovarian estrogen production. The second control group included 27 randomly selected consecutive patients with endometrial carcinoma without known history of tamoxifen use or pathological evidence of ovarian hyperthecosis or other ovarian estrogen-producing lesions. A total of 1 to 39 (mean 20) hematoxylin-eosin-stained slides were reviewed for each case and the endometrial carcinomas were classified based on the WHO classification. ${ }^{34}$ Ovarian hyperthecosis was defined as the presence of luteinized stromal cells in the ovarian parenchyma and was classified as mild (scattered cells); moderate (small nests of luteinized stromal cells) and extensive (nodules of luteinized stromal cells). Limited clinical information was available for all patients. Since the study was retrospective, laboratory values for estrogen levels could not be obtained. IRB approval was obtained from Partners Healthcare System.

\section{Immunohistochemistry for $\beta$-catenin}

Immunohistochemistry for $\beta$-catenin was performed on paraffin-embedded tissue sections, using heatinduced antigen retrieval before exposure to the primary antibody. Mouse antihuman $\beta$-catenin monoclonal antibody (Transduction Laboratories, Lexington, KY) was applied at 1:1000 dilution. Immunodetection was performed with biotinylated antimouse immunoglobulins and peroxidase-labeled streptavidin (LSAB-DAKO, Glostrup, Denmark) with diaminobenzidine as chromogen. Nuclear immunostaining of neoplastic epithelial component was considered to be positive, irrespective of the percentage of stained nuclei. Fifty-nine paraffinembedded tissue blocks with sufficient quantity of malignant cells were available for immunohistochemical study.

\section{Mutational Analysis}

Samples were manually microdissected to ensure $>75 \%$ of neoplastic cells. DNA was extracted with phenol/chloroform after proteinase $\mathrm{K}$ digestion. We 
screened the phosphorylation sequence of GSK- $3 \beta$ in $\beta$-catenin, codons 12 and 13 of $K$-ras and the complete coding sequence of PTEN for mutations. ${ }^{35}$ PCR amplification products were subjected to direct sequencing to analyze mutations in $\beta$-catenin and K-ras. To analyze PTEN mutations, PCR products were subjected to heteroduplex analysis by conformation-sensitive gel electrophoresis. PTEN gene mutations were analyzed using primers described previously. ${ }^{36}$ Amplified samples were held at $95{ }^{\circ} \mathrm{C}$ for $5 \mathrm{~min}$ and at $65^{\circ} \mathrm{C}$ for $1 \mathrm{~h}$ to generate heteroduplexes. The products were diluted 1:2 in sucrose buffer and loaded onto a partially denaturing MDE gel at constant $7 \mathrm{~W}$ power. Gels were silver stained and dried on a vacuum gel dryer. Cases showing heteroduplex formation were sequenced. The BigDye Sequencing Kit (Applied Biosystems, Foster City, CA) was used for sequencing the PCR products. Product reactions were run in an Applied Biosystems 3700 Genetic Analyzer (Applied Biosystems). Sequencing was performed in both directions with the primer used for PCR. To determine MSI, we analyzed two mononucleotide repeats, BAT-26 and $B A T-25$. Primers, PCR amplification conditions and PCR product analysis were made as described previously. ${ }^{35}$ We considered a phenotype to be replication error positive when the tumors had deletions of more than $2 \mathrm{bp}$ in BAT 26 and BAT 25 . Fifty-nine paraffin-embedded tissue blocks with sufficient quantity of malignant cells were available for molecular analysis.

\section{Statistical Analysis}

The statistical significance of the differential immunohistochemical and molecular profile in endometrial carcinoma in patients with history of tamoxifen treatment, and the two control groups of endometrial carcinoma was assessed using $\chi^{2}$ test of Pearson. A probability of $P<0.05$ was considered statistically significant. Only pure endometrioid endometrial carcinomas were included in the statistical analysis to assure that similar lesions were compared in all groups and statistical results were valid and reproducible.

\section{Results}

\section{Clinicopathologic Findings}

Sixty-one patients with endometrial carcinoma were studied. The clinicopathologic findings of patients with history of tamoxifen use are summarized in Table 1. Findings for control patients are presented in Table 2. Overall, patients ranged in age from 39 to 84 (mean 63) years.

Eighteen patients in the tamoxifen group ranged in age from 48 to 84 (mean 64) years. All had a history of breast cancer. Tamoxifen therapy lasted from 6 months to 6 (average 4) years. Endometrial carcinoma developed from 6 months to 25 (mean 8.5) years after the detection of the breast cancer. In six patients (40\%), endometrial carcinoma was detected while they were on tamoxifen treatment. Four patients had additional primary malignancies: lung cancer (1), colon cancer (2) and bladder and lung cancer (1). According to the FIGO classification, there were 15 stage I (10 IA, four IB and one IC); one stage II and two stage III ECs. Sixteen were endometrioid endometrial carcinomas, one was a clear cell carcinoma and one a mixed endometrioid and serous carcinoma. This last patient had a recurrence in the abdominal wall and was treated with chemotherapy. A patient with an endometrioid endometrial carcinoma grade $3 / 3$, stage III received multiple courses of chemotherapy following the hysterectomy and was alive with metastatic carcinoma after 4 years. All the other patients were treated with surgery alone. Thirteen patients were alive and well after a mean follow-up of 7.6 (range 4-18) years. One patient died of the disease, one patient had stable metastatic disease in the peritoneal cavity and three patients were lost to follow-up. Carcinoma formed a well-circumscribed mass in eight cases, ranging from $1.6-4 \mathrm{~cm}$ (mean 2.6), while in three cases, the tumor was diffuse. Two endometrial carcinomas were confined to an endometrial polyp and in four cases the endometrial carcinoma was a microscopic finding. Histologically, there were 16 endometrioid endometrial carcinomas (11 grade 1, three grade 2 and two grade 3 ), four of them with squamous differentiation (Figure 1a). In eight cases, carcinoma coexisted with endometrial hyperplasia (complex hyperplasia with atypia (7) and simple hyperplasia without atypia (1)).

Sixteen patients with ovarian hyperthecosis and adult granulosa cell tumor ranged in age from 39 to 84 (mean 60) years. The patient with adult granulosa cell tumor was 65 years old. According to the FIGO staging classification, there were 12 stage I (eight IA, three IB and one IC), two stage II and two stage III endometrial carcinomas. The patient with stage IC and both patients with stage III EC received radiotherapy. One patient with stage III endometrial carcinoma (extensive lymph node metastases) received additional chemotherapy. All other patients were treated with surgical resection only. Twelve patients were alive and well after a mean follow-up of 4.0 (range 2-6) years. Four patients, including the patient with adult granulosa cell tumor, were lost to follow-up. On pathologic examination, the tumor formed a well-circumscribed mass in eight cases, ranging from $0.6-5 \mathrm{~cm}$ (mean 2.7 ) and was diffusely involving the endometrium in seven cases. All patients had endometrioid endometrial carcinoma (11 grade 1 and four grade 2), six of them with squamous differentiation (Figure 2a). In two patients, the endometrioid endometrial carcinoma coexisted with complex atypical endometrial hyperplasia. Among 
Table 1 Clinical features of patients with endometrial carcinoma and tamoxifen exposure

\begin{tabular}{|c|c|c|c|c|c|c|c|c|c|}
\hline No. & Age & $T$ & $I B C A-E C$ & $\begin{array}{l}\text { EC type, } \\
\text { grade }\end{array}$ & Size & Myo inv & Mets & $T X$ & Survival \\
\hline 1 & 66 & $\mathrm{U}$ & 7 years & EEC, $1 / 3$ & $2 \mathrm{~cm}$ & No & No & No & 18 years \\
\hline 2 & 79 & 19 years & 19 years & CCA, $2 / 3$ & Diffuse & $\begin{array}{l}\text { Inner } 1 / 2 \text { of } \\
\text { myometrium }\end{array}$ & Ovaries, LNs & $\mathrm{U}$ & $\mathrm{U}$ \\
\hline 3 & 63 & 4 years & 15 years & EEC, $1 / 3$ & $1.5 \mathrm{~cm}$ & $\begin{array}{l}\text { Inner } 1 / 2 \text { of } \\
\text { myometrium }\end{array}$ & No & No & $\mathrm{U}$ \\
\hline 4 & 74 & 3 years & 25 years & EEC, $1 / 3$ & $4 \mathrm{~cm}$ & $\begin{array}{l}\text { Inner } 1 / 2 \text { of } \\
\text { myometrium }\end{array}$ & No & No & 9 years \\
\hline 5 & 54 & 6 years & 11 years & EEC, $1 / 3$ & $2.6 \mathrm{~cm}$ & No & No & No & $\mathrm{U}$ \\
\hline 6 & 71 & 1 years & 1 year & EEC, $1 / 3$ & $\mathrm{U}$ & No & No & No & 6 years \\
\hline 7 & 84 & 3 years & 6 years & $\begin{array}{l}\text { EEC+SPC, } \\
3 / 3\end{array}$ & Diffuse & No & No & Chemo & 5 years \\
\hline 8 & 64 & 4 years & 7 years & EEC, $1 / 3$ & MF & No & No & No & 5 years \\
\hline 9 & 60 & $\mathrm{U}^{\circ}$ & 10 years & EEC, $3 / 3$ & $3 \mathrm{~cm}$ & Uterine serosa & $\begin{array}{l}\text { Both ovaries and } \\
\text { tubes, omentum }\end{array}$ & Chemo & $\begin{array}{l}4 \text { years, alive } \\
\text { with disease }\end{array}$ \\
\hline 10 & 77 & 6 months & 6 months & EEC, $3 / 3$ & $4 \mathrm{~cm}$ & $\begin{array}{l}\text { Inner } 1 / 2 \text { of } \\
\text { myometrium }+\end{array}$ & LN, bone & No & $\begin{array}{l}3 \text { years, death } \\
\text { from EC }\end{array}$ \\
\hline 11 & 71 & 5 years & 6 years & EEC, $1 / 3$ & $1.6 \mathrm{~cm}$ & No & No & No & 5 years \\
\hline 12 & 60 & 4 years & 4 years & $\mathrm{EEC}, 2 / 3$ & $\mathrm{U}$ & $\begin{array}{l}\text { Inner } 1 / 2 \text { of } \\
\text { myometrium }\end{array}$ & No & No & 4 years \\
\hline 13 & 53 & 5 years & 5 years & EEC, $1 / 3$ & $\mathrm{MF}$ & No & No & No & 4 years \\
\hline 14 & 75 & 6 years & 6 years & EEC, $2 / 3$ & $2.5 \mathrm{~cm}$ & $\begin{array}{l}\text { Outer } 1 / 2 \text { of } \\
\text { myometrium }\end{array}$ & No & No & 8 years \\
\hline 15 & 69 & 5 years & 5 years & EEC, $1 / 3$ & $\mathrm{MF}$ & No & No & No & 8 years \\
\hline 16 & 48 & $\mathrm{U}$ & U & EEC, $1 / 3$ & Diffuse & No & No & No & 6 years \\
\hline 17 & 48 & $\mathrm{U}$ & $\mathrm{U}$ & EEC, $2 / 3$ & $2.8 \mathrm{~cm}$ & $\begin{array}{l}\text { Inner } 1 / 2 \text { of } \\
\text { myometrium }\end{array}$ & No & No & 4 years \\
\hline 18 & 64 & $\mathrm{U}$ & $\mathrm{U}$ & EEC, $1 / 3$ & $\mathrm{U}$ & No & No & No & 7 years \\
\hline
\end{tabular}

CCA, clear cell carcinoma; EC, endometrial carcinoma; EEC, endometrioid endometrial carcinoma; IBCA-EC, interval between breast cancer and development of EC; MF, microscopic foci; Myo inv, myometrial invasion; SPC, serous papillary carcinoma; T, duration of treatment with tamoxifen; TX, adjuvant treatment; U, unknown.

Table 2 Clinical features of patients with endometrial carcinoma related to ovarian hyperthecosis (cases 1-15) and adult granulosa cell tumor of the ovary (case 16).

\begin{tabular}{|c|c|c|c|c|c|c|c|c|}
\hline \multirow[t]{2}{*}{ No. } & \multirow[t]{2}{*}{ Age } & \multirow[t]{2}{*}{$\mathrm{OH}$} & \multicolumn{4}{|c|}{$E E C$} & \multirow[t]{2}{*}{$T X$} & \multirow[t]{2}{*}{ Surviva } \\
\hline & & & Size & Grade & Myo inv & Extraut ext & & \\
\hline 1 & 53 & ++ & Diffuse & $1 / 3$ & Inner $1 / 2$ & No & No & 5 years \\
\hline 2 & 61 & ++ & $5 \mathrm{~cm}$ & $1 / 3$ & No & No & No & $\mathrm{U}$ \\
\hline 3 & 62 & ++ & Diffuse & $1 / 3$ & No & No & No & 4 years \\
\hline 4 & 64 & ++ & Diffuse & $2 / 3$ & No & No & No & 3 years \\
\hline 5 & 72 & ++ & $2.2 \mathrm{~cm}$ & $1 / 3$ & No & No & No & 4 years \\
\hline 6 & 51 & + & $2.5 \mathrm{~cm}$ & $2 / 3$ & No & No & No & 4 years \\
\hline 7 & 48 & ++ & $0.6 \mathrm{~cm}$ & $1 / 3$ & No & No & No & 4 years \\
\hline 8 & 55 & + & $1.4 \mathrm{~cm}$ & $1 / 3$ & Inner $1 / 2$ & No & No & 3 years \\
\hline 9 & 84 & +++ & $\mathrm{U}$ & $1 / 3$ & No & No & No & 1year \\
\hline 10 & 58 & ++ & Diffuse & $2 / 3$ & Inner $1 / 2$ & No & No & U \\
\hline 11 & 54 & ++ & $3.0 \mathrm{~cm}$ & $1 / 3$ & No & No & No & 2 years \\
\hline 12 & 39 & ++ & Multiple microfoci & $1 / 3$ & No & No & No & $\mathrm{U}$ \\
\hline 13 & 63 & ++ & $3.1 \mathrm{~cm}$ & $2 / 3$ & Inner $1 / 2$ & 6/23 LNs & Chemo, RXT & 3 years \\
\hline 14 & 67 & + & $4.4 \mathrm{~cm}$ & $1 / 3$ & Outer $1 / 2$ & 1/19 LNs & $\mathrm{RXT}$ & 2 years \\
\hline 15 & 60 & +++ & Diffuse & $1 / 3$ & Outer $1 / 2$ & No & RXT & 2 years \\
\hline 16 & 65 & No & Diffuse & $1 / 3$ & No & No & No & $\mathrm{U}$ \\
\hline
\end{tabular}

OH, ovarian hyperthecosis; Chemo, RXT, chemotherapy, radiation treatment; EEC, endometrioid endometrial carcinoma; Extraut ext, extrauterine extension; LN, lymph node; Myo inv, myometrial invasion; TX, adjuvant treatment; U, unknown.

the patients selected into the ovarian hyperthecosis group, three patients had mild ovarian hyperthecosis, 10 patients had moderate ovarian hyperthecosis and two had extensive ovarian hyperthecosis (Figure 2b).
Twenty-seven patients in the randomly selected control group ranged in age from 50 to 79 (mean 64) years. Twenty-three patients had stage I endometrial carcinoma (four IA, 18 IB and one IC); two stage II endometrial carcinoma (one IIA and one IIB) and 


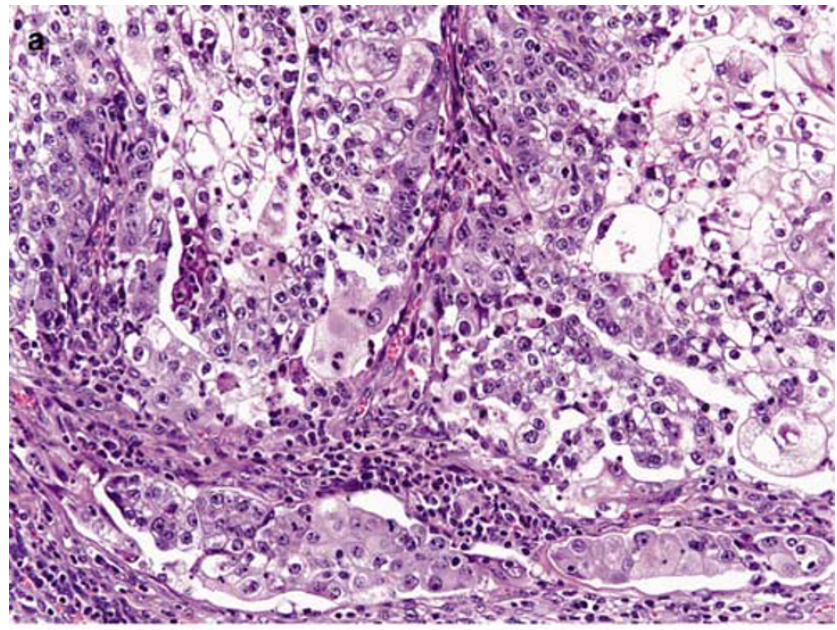

b
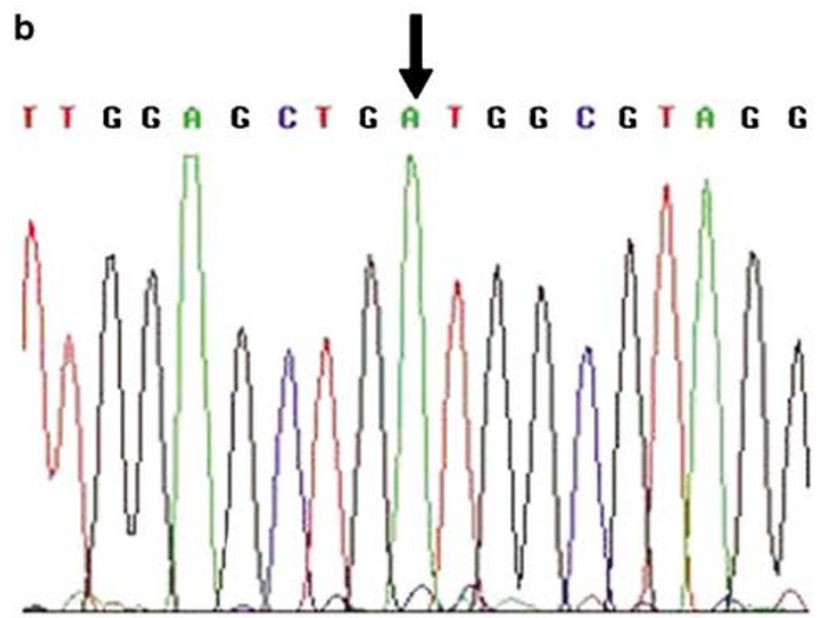

c
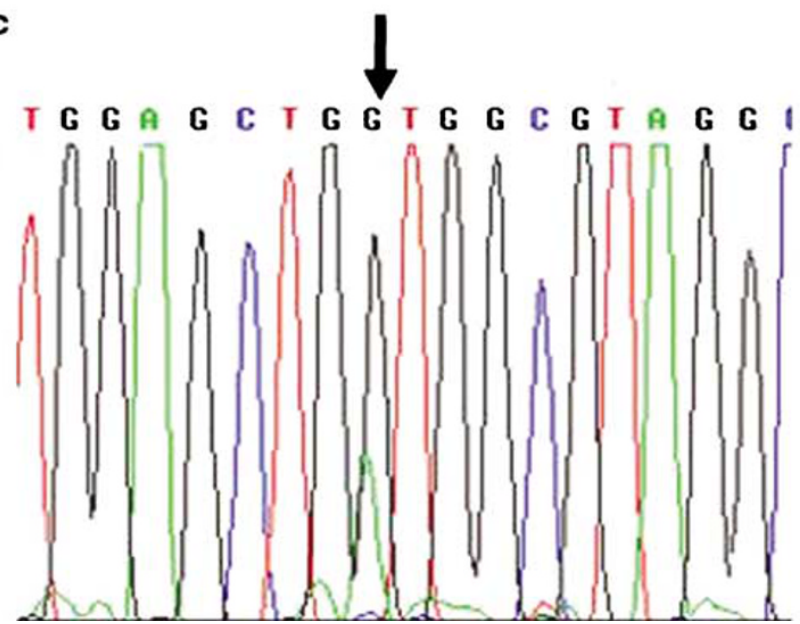

Figure 1 Patient with history of tamoxifen exposure (case 7). (a) A moderately differentiated endometrioid endometrial carcinoma. (b) K-ras Gly(GGT)12(GAT)Asp mutation compared to (c) K-ras wild type.

one had a IIIA endometrial carcinoma. One patient had a carcinosarcoma with lymph node metastasis (stage IIIC). Two endometrial carcinomas had local recurrence to the vaginal apex, but all patients were alive and well after a mean follow-up of 4.2 (range
3-6) years. The tumors formed a well-circumscribed mass in 21 cases, ranging from $1.5-5.5 \mathrm{~cm}$ (mean 3.4 ) and diffusely involved the endometrium in six cases. Twenty-six patients had endometrioid endometrial carcinoma (17 grade 1 , six grade 2 and three grade 3 ), three with squamous differentiation and three associated with atypical endometrial hyperplasia.

\section{Immunohistochemical Findings}

The immunohistochemical results are described in Tables 3-5 and summarized in Table 6. Immunohistochemical analysis for $\beta$-catenin was performed on 59 tumors, 54 of them being informative. Two cases in the tamoxifen group were excluded due to technical difficulties. Nuclear staining was seen in 12 of 54 endometrial carcinomas (22\%), with 4/11 in the tamoxifen group (36\%), 7/16 in the ovarian hyperthecosis/adult granulosa cell tumor group (44\%), including the patient with history of adult granulosa cell tumor and 1/27 in the random control group ( $4 \%$; Figure 2c). The $\beta$-catenin immunopositivity was significantly higher in both tamoxifen and ovarian hyperthecosis/adult granulosa cell tumor groups as compared to the random controls $(P=0.004)$.

\section{Molecular Findings}

The molecular results are described in Tables 3-5 and summarized in Table 6 . Nine of 59 available cases were excluded from the final analysis because of technical difficulties (5), absence of tissue (1) and mixed histomorphology (3).

Mutations in $\beta$-catenin gene were identified in $8 / 52$ informative cases (15\%), including three endometrial carcinomas in the tamoxifen group (30\%), four in the ovarian hyperthecosis/adult granulosa cell tumor group $(22 \%$, one of them in adult granulosa cell tumor patient) and one random control endometrial carcinoma (4\%; Figure $2 \mathrm{~d}$ and e). All positive cases had concomitant nuclear staining for $\beta$-catenin. The higher prevalence of $\beta$-catenin mutations in patients with history of tamoxifen use and ovarian hyperthecosis/adult granulosa cell tumor as opposed to patients in the random control group was statistically significant $(P=0.05)$.

$K$-ras mutations were found in 6/57 informative endometrial carcinomas (11\%), being present in three endometrial carcinomas in the tamoxifen group $(21 \%)$, one in the ovarian hyperthecosis/ adult granulosa cell tumor group $(6 \%)$ and two random controls (7\%; Figure 1b and c). One positive endometrial carcinoma in the tamoxifen group was a grade 3 mixed carcinoma; it was excluded from the statistical analysis. K-ras mutations appeared to be more common among endometrial carcinomas in patients with history of 

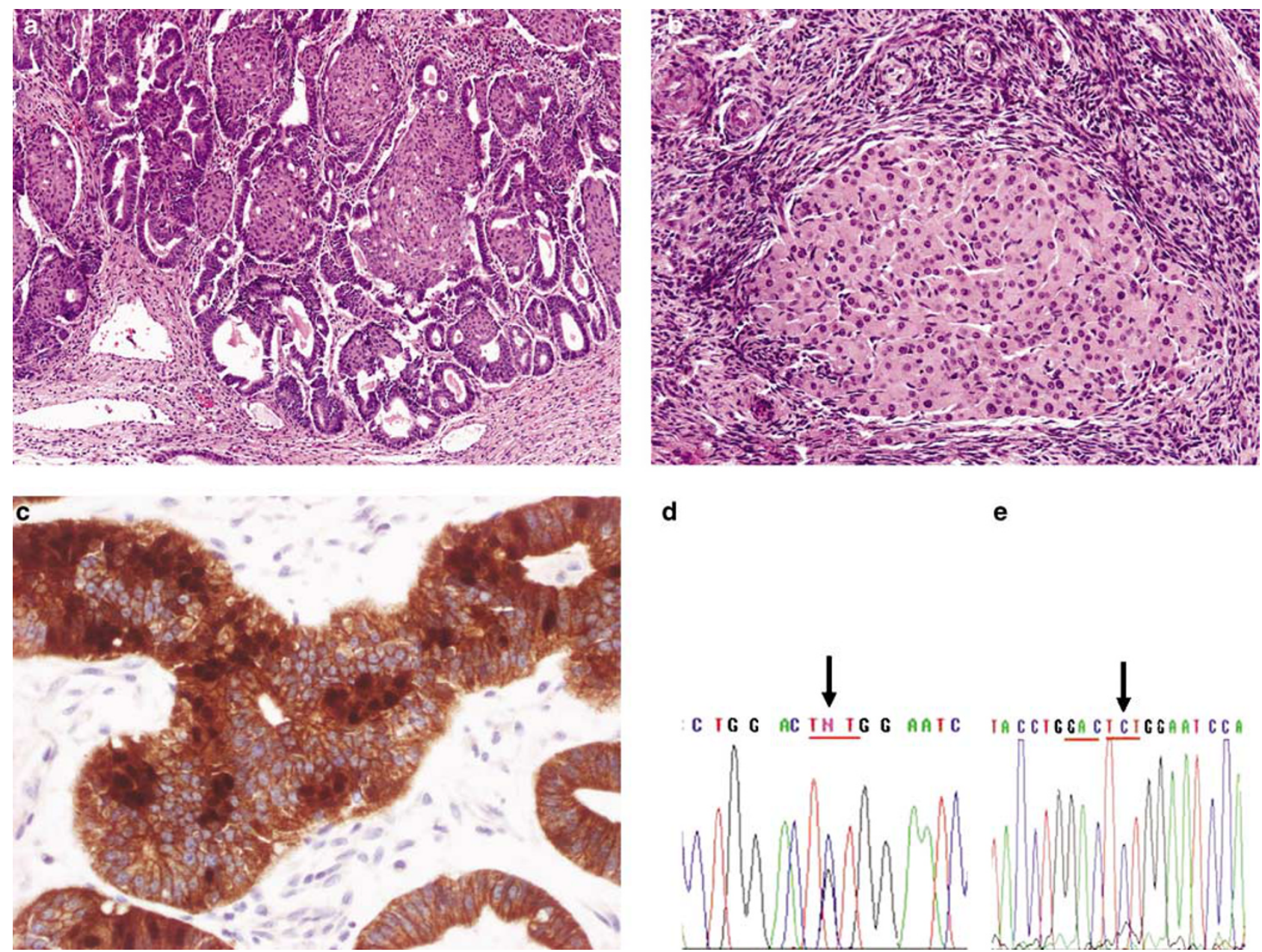

d

e

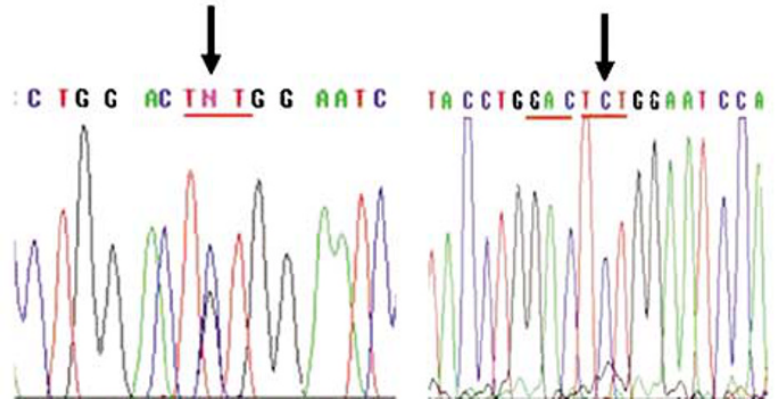

Figure 2 Patient with ovarian hyperthecosis (case 1). (a) A well-differentiated endometrioid endometrial carcinoma is associated with extensive morular formation. (b) Large collection of luteinized cells is present in the background ovarian stroma. (c) Immunostaining for $\beta$-catenin with neoplastic glands showing focal nuclear positivity. (d) $\beta$-catenin Ser(TCT)37(TTT)Phe mutation compared to (e) $\beta$-catenin wild type.

Table 3 Immunohistochemistry and mutational analyses of endometrial carcinomas associated with tamoxifen

\begin{tabular}{|c|c|c|c|c|c|c|c|c|}
\hline \# & Age & EC type, grade & EC stage & $\beta$-catenin, nuclear IHC & $\beta$-catenin mutation & MSI & PTEN & $K$-ras mutation \\
\hline 1 & 66 & EEC, $1 / 3$ & IA & Pos & Asp(GAC)32(TAC)Tyr & - & - & - \\
\hline 2 & 79 & CCC, $2 / 3$ & III & - & - & - & - & - \\
\hline 3 & 63 & EEC, $1 / 3$ & IB & - & - & - & - & - \\
\hline 4 & 74 & EEC, $1 / 3$ & IA & Pos & Ser(TCT)33(TGT)Cys & - & - & - \\
\hline 5 & 54 & EEC, $1 / 3$ & IA & - & - & - & - & Gly(GGT)12(GAT)Asp \\
\hline 6 & 71 & EEC, $1 / 3$ & IA & - & NR & - & - & - \\
\hline 7 & 84 & MC, $3 / 3$ & IA & - & - & - & - & Gly(GGT)12(GAT)Asp \\
\hline 8 & 64 & EEC, $1 / 3$ & IA & - & NR & - & - & - \\
\hline 9 & 60 & EEC, $3 / 3$ & III & - & - & - & - & - \\
\hline 10 & 77 & EEC, $3 / 3$ & II & Pos & - & - & - & - \\
\hline 11 & 71 & EEC, $1 / 3$ & IA & - & - & - & - & - \\
\hline 12 & 60 & EEC, $2 / 3$, & IB & - & - & - & - & - \\
\hline 13 & 53 & EEC, $1 / 3$ & IB & NR & NR & - & - & NR \\
\hline 14 & 75 & $\mathrm{EEC}, 2 / 3$ & IC & - & - & - & $\operatorname{Pos}^{a}$ & - \\
\hline 15 & 69 & EEC, $1 / 3$ & IA & NR & NR & - & - & NR \\
\hline 16 & 48 & EEC, $1 / 3$ & IA & - & - & Pos & - & - \\
\hline 17 & 48 & EEC, $2 / 3$ & IB & - & - & - & - & Gly(GGC)13(TGC)Cys \\
\hline 18 & 64 & $\mathrm{EEC}, 1 / 3$ & IA & Pos & Ser(TCT)33(TGT)Cys & - & - & - \\
\hline
\end{tabular}

CCC, clear cell carcinoma; EC, endometrial carcinoma; MC, mixed carcinoma; NR, not reported.

${ }^{a}$ PTEN mutation case 14: Arg(CGA)130(TGA)Stop E5 and exon 4+1 G to A (splicing) E4. 
Table 4 Immunohistochemistry and mutational analyses of endometrial carcinomas associated with ovarian hyperthecosis (cases 1-15) and adult granulosa cell tumor (case 16)

\begin{tabular}{|c|c|c|c|c|c|c|c|c|}
\hline \# & Age & EC type, grade & EC stage & $\beta$-catenin, nuclear IHC & $\beta$-catenin mutation & $M S I$ & $P T E N^{\mathrm{a}}$ & K-ras mutation \\
\hline 1 & 53 & $1 / 3$ & IB & Pos & Ser(TCT)37(TTT)Phe & - & - & - \\
\hline 2 & 61 & $1 / 3$ & IA & - & - & Pos & - & - \\
\hline 3 & 62 & $1 / 3$ & II & - & - & - & - & - \\
\hline 4 & 64 & $2 / 3$ & II & Pos & - & Pos & - & - \\
\hline 5 & 72 & $1 / 3$ & IA & Pos & NI & - & - & - \\
\hline 6 & 51 & $2 / 3$ & IA & Pos & Ser(TCT)37(TTT)Phe & - & Pos & - \\
\hline 7 & 48 & $1 / 3$ & IA & - & - & - & - & Gly(GGT)12(GAT)Asp \\
\hline 8 & 55 & $1 / 3$ & IB & - & - & - & - & - \\
\hline 9 & 84 & $1 / 3$ & IA & - & - & - & - & - \\
\hline 10 & 58 & $2 / 3$ & IB & Pos & Gly(GGA)34(GTA)Val & - & Pos & - \\
\hline 11 & 54 & $1 / 3$ & IA & - & - & - & - & - \\
\hline 12 & 39 & $1 / 3$ & IA & Pos & - & - & - & - \\
\hline 13 & 63 & $2 / 3$ & III & - & - & Pos & Pos & - \\
\hline 14 & 67 & $1 / 3$ & III & - & - & - & Pos & - \\
\hline 15 & 60 & $1 / 3$ & IC & - & - & - & Pos & - \\
\hline 16 & 65 & $1 / 3$ & IA & Pos & Del13ntcodon30 & - & - & - \\
\hline
\end{tabular}

aPTEN mutations: case 6: exon 4-7 del_CTTTT (splicing) E4; case 10, 13 and 14: Arg(CGA)130(GGA)Gly E5; case 15: 344 del_A E5.

Table 5 Immunohistochemistry and mutational analysis of endometrial carcinomas in random control group

\begin{tabular}{|c|c|c|c|c|c|c|c|c|}
\hline No. & Age & EC type, grade & EC stage & $\beta$-catenin, nuclear IHC & $\beta$-catenin mutation & $M S I$ & $P T E N^{a}$ & K-ras mutation \\
\hline 1 & 57 & EEC, $3 / 3$ & IIIA & - & - & - & - & - \\
\hline 2 & 73 & EEC, $1 / 3$ & IB & - & - & - & - & - \\
\hline 3 & 79 & EEC, $1 / 3$ & IB & Pos & Ser(TCT)33(TGT)Cys & - & - & - \\
\hline 4 & 62 & EEC, $1 / 3$ & IA & - & - & - & Pos & - \\
\hline 5 & 64 & EEC, $1 / 3$ & IB & - & - & - & - & Gly(GGT)12(GTT)Val \\
\hline 6 & 65 & EEC, $2 / 3$ & IB & - & - & - & - & - \\
\hline 7 & 75 & EEC, $1 / 3$ & IB & - & - & Pos & - & - \\
\hline 8 & 64 & EEC, $1 / 3$ & IB & - & - & Pos & Pos & - \\
\hline 9 & 76 & EEC, $2 / 3$ & IB & - & - & Pos & - & - \\
\hline 10 & 68 & EEC, $1 / 3$ & IB & - & - & NR & Pos & - \\
\hline 11 & 76 & EEC, $3 / 3$ & IB & - & - & NR & - & - \\
\hline 12 & 57 & EEC, $1 / 3$ & IB & - & - & - & Pos & - \\
\hline 13 & 52 & EEC, $2 / 3$ & IB & - & - & - & - & - \\
\hline 14 & 52 & EEC, $2 / 3$ & IB & - & - & Pos & Pos & - \\
\hline 15 & 75 & EEC, $1 / 3$ & IB & - & - & - & Pos & - \\
\hline 16 & 69 & EEC, $2 / 3$ & IB & - & - & - & Pos & Gly(GGT)12(GTT)Val \\
\hline 17 & 68 & EEC, $1 / 3$ & IC & - & - & - & - & - \\
\hline 18 & 65 & EEC, $1 / 3$ & IB & - & - & Pos & - & - \\
\hline 19 & 59 & EEC, $1 / 3$ & IB & - & - & Pos & Pos & - \\
\hline 20 & 66 & $\mathrm{EEC}, 3 / 3$ & IB & - & - & Pos & Pos & - \\
\hline 21 & 58 & EEC, $1 / 3$ & IA & - & - & - & - & - \\
\hline 22 & 56 & EEC, $1 / 3$ & IIA & - & - & - & Pos & - \\
\hline 23 & 54 & EEC, $2 / 3$ & IB & - & - & Pos & Pos & - \\
\hline 24 & 56 & EEC, $1 / 3$ & IA & - & - & - & Pos & - \\
\hline 25 & 79 & $\mathrm{CS}$ & IIIC & - & - & - & - & - \\
\hline 26 & 56 & EEC, 1/3 & IIB & - & - & NR & - & - \\
\hline 27 & 50 & EEC, $1 / 3$ & IA & - & - & NR & - & - \\
\hline
\end{tabular}

CS, carcinosarcoma; EEC, endometrioid endometrial carcinoma; NR, not reported.

aPTEN mutations: case 4: 469_470ins G E5, Arg(CGA)233(TGA)Stop E7; case 8: 330_331delA E5; case 10: 662_663delA E7; case 12: 405_406insA E5; case 14: Tyr(TAC)65(TAA)Stop E3, Arg(CGA)130(TGA)Stop E5; case 15: 932_933insA E8; case 16: 955 958delACTT E8; case 19: 783_784delGA E7; case 20: Arg(CGA)130(TGA)Stop E5, Arg(CGA)233(TGA)Stop E7; case 22: 723_724insT E7; case 23: 170_171insT E3, 801_802 ins A E7; case 24: $\operatorname{Arg}(\mathrm{CGA}) 130(\mathrm{GGA})$ Gly E5.

tamoxifen exposure and were comparably low in both control groups; however, the difference was not statistically significant.

Mutations in PTEN were noted in 18 of 54 endometrial carcinomas (33\%). The highest incidence was found in the random control group with
12 of 27 positive cases (44\%), followed by the ovarian hyperthecosis/adult granulosa cell tumor group with five positive cases (31\%). Only one positive endometrial carcinoma was present in the tamoxifen group (9\%). Even though there was a decreased frequency of PTEN mutations in endo- 
Table 6 Summary of immunohistochemical and molecular profiles in all groups

\begin{tabular}{|c|c|c|c|c|c|}
\hline & Tamoxifen $^{\mathrm{a}}$ & $O H / A G C T^{\mathrm{a}}$ & Random control ${ }^{\mathrm{a}}$ & Both controls & Total \\
\hline$\beta$-catenin immunoexpression ${ }^{\mathrm{b}}$ & $4 / 11(36 \%)$ & $7 / 16(44 \%)$ & $1 / 27(4 \%)$ & $8 / 43(19 \%)$ & $12 / 54(22 \%)$ \\
\hline$\beta$-catenin mutation ${ }^{\mathrm{b}}$ & $3 / 10(30 \%)$ & $4 / 15(27 \%$ & $1 / 27(4 \%)$ & $5 / 42(12 \%)$ & $8 / 52(15 \%)$ \\
\hline K-ras mutation & $3 / 14(21 \%)$ & $1 / 16(6 \%)$ & $2 / 27(7 \%)$ & $3 / 43(7 \%)$ & $6 / 57(11 \%)$ \\
\hline MSI & $1 / 12(8 \%)$ & $3 / 16(19 \%)$ & $8 / 22(36 \%)$ & $11 / 38(29 \%)$ & $12 / 50(24 \%)$ \\
\hline PTEN mutation & $1 / 11(9 \%)$ & $5 / 16(31 \%)$ & $12 / 27(44 \%)$ & $17 / 43(40 \%)$ & $18 / 54(33 \%)$ \\
\hline
\end{tabular}

OH/AGCT, ovarian hyperthecosis/adult granulosa cell tumor.

${ }^{\mathrm{a}}$ Groups compared by statistical analysis.

bIndicates a statistically significant result.

metrial carcinoma from patients with tamoxifen exposure as opposed to the control groups, the results were not statistically significant.

MSI was found in 12 of 50 endometrial carcinomas $(24 \%)$. Eight of 22 analyzed endometrial carcinomas showed MSI (36\%) in the random control group, followed by the ovarian hyperthecosis/adult granulosa cell tumor group with three cases $(19 \%)$ and one MSI case in the tamoxifen group $(8 \%)$. The differences were not statistically significant.

\section{Discussion}

The exact nature of the carcinogenic effect of tamoxifen on endometrial cells is not well understood. On the basis of the crystal structures of ligand bound hormone-binding domains of estrogen receptor, it is believed that tamoxifen acts as an estrogen receptor antagonist in the breast tissue, binding to the estrogen receptor and inducing a conformational change that blocks its interaction with coactivation proteins. ${ }^{27,28}$ However, this mechanism does not explain the weak estrogenic effect that tamoxifen exerts in the endometrium. The genotoxic theory is based on the fact that tamoxifen forms a DNA adduct, which has been detected in half of endometrial samples obtained from women treated with the drug. ${ }^{37}$ This compound has been shown to induce mutations in simian cells; however, its significance in human endometrium is still unclear. ${ }^{38}$ Another hypothesis is based on the estrogen receptor-dependent model. Studies indicate that tamoxifen binds to estrogen receptor but may have a distinct genomic activity from estrogen. ${ }^{26}$ It is possible that estrogen-liganded and tamoxifenliganded estrogen receptors possess different affinities for different gene promoters and thus regulate different sets of genes. ${ }^{27,28}$ In vivo, the relationship between exposure to tamoxifen and differential expression of molecular markers implicated in the pathogenesis of endometrial carcinoma has not been well established. To address this question, we selected a group of patients with history of tamoxifen use who subsequently developed endometrial carcinoma and compared it to a control group without tamoxifen use. To explore the relationship between tamoxifen-induced carcinogenesis and estrogen-induced carcinogenesis, we divided the control group into two. Some patients were chosen because they had histological evidence of ovarian hyperthecosis or adult granulosa cell tumor of the ovary-conditions associated with increased levels of endogenous estrogens. Ovarian hyperthecosis is characterized by a proliferation of luteinized cells with abundant androgen-containing granules. These androgens undergo peripheral conversion to a weaker form of estrogen, estrone. A patient with adult granulosa cell tumor of the ovary was chosen because of estrogen production by these tumors and their known association with endometrial carcinoma. Another control group of patients with endometrial carcinoma were randomly selected, assuming they were previously healthy females with no history of tamoxifen exposure or hyperestrogenism.

The main and novel finding of our study is the evidence of increased rate of $\beta$-catenin abnormalities in patients with tamoxifen use as compared with random controls. By immunohistochemistry, nuclear staining for $\beta$-catenin was detected in four out of 11 informative cases with history of tamoxifen exposure $(36 \%)$ and only one out of 27 patients in the random control group (4\%), a statistically significant result $(P=0.004)$. The rate of $\beta$-catenin mutations also was significantly increased in patients in the tamoxifen group as opposed to the random control group (30 vs $4 \% ; P=0.05$ ). Interestingly, both nuclear overexpression and the rate of $\beta$-catenin mutations were similar in the group of ovarian hyperthecosis/adult granulosa cell tumor and in the tamoxifen group (44 vs $36 \%$ and $27 \mathrm{vs}$ $30 \%$, respectively). These findings suggest that the carcinogenic effect of tamoxifen is, at least in part, due to its agonistic estrogenic effect through estrogen receptor binding.

Estrogen stimulation has been found to directly upregulate the CTNNB1 gene, inducing accumulation and nuclear localization of $\beta$-catenin. ${ }^{39,40}$ Studies in vitro suggest a possible link between the estrogen-signaling pathway and the Wnt-signaling pathway as $\beta$-catenin may form a complex with steroid hormone receptors, such as the androgen 
receptor and the retinoic acid receptor, modulating receptor-dependent transcriptional activity. ${ }^{41,42}$ Estrogen has also been shown to induce upregulation of Wnt proteins in a breast cancer cell line. ${ }^{40}$ In a gene expression profile study of tamoxifen treatment effects on the endometrium, $\beta$-catenin emerged as one of five differentially expressed genes. ${ }^{43}$

All cases with $\beta$-catenin mutations had a concomitant nuclear overexpression of $\beta$-catenin; however, some cases with immunohistochemical positivity for $\beta$-catenin did not show evidence of $\beta$-catenin mutations by PCR sequencing analysis (overall there were 12 cases with positive nuclear staining, of which eight had proven $\beta$-catenin mutations). This discrepancy may be explained by the fact that $\beta$-catenin can be localized to both cytoplasm and nucleus of endometrial cells. The cytoplasmic $\beta$-catenin is low in normal cells since the protein is rapidly degraded by adenomatous polyposis coli (APC) protein together with glycogen synthetase kinase-3b (GAK-3b). Mutations of $\beta$-catenin increase cytoplasmic levels of free $\beta$-catenin, which translocates to the cell nucleus and then acts as an oncoprotein through the formation of complexes with the DNA-binding proteins of the T-cell factor-lymphoid enhancer factor family. ${ }^{8}$

According to the literature, complex endometrial hyperplasia with immature squamous metaplasia (squamous morules) may be associated with $\beta$-catenin mutations and better clinical outcome. ${ }^{44}$ Our study supports these results as seven of 13 patients (54\%) with endometrial carcinoma and areas of squamous differentiation had evidence of $\beta$-catenin mutations. By contrast, only one of 48 patients $(2 \%)$ without squamous morules had a $\beta$-catenin mutation. Furthermore, six of eight patients had $\beta$-catenin mutation as a sole molecular abnormality and presented with low grade (grade 1/3) and low stage (stage I) tumors with endometrioid morphology. The remaining two of eight patients with $\beta$-catenin mutations had a concomitant PTEN mutation and slightly higher grade tumors (grade 2 of three), although they were still stage I endometrioid endometrial carcinomas. One patient with nuclear overexpression and unmutated $\beta$-catenin also had MSI; she presented with a grade 2, stage II endometrioid endometrial carcinoma. All three patients with combined molecular defects had ovarian hyperthecosis; but its degree did not seem to correlate with the presence of molecular alterations, in particular, the presence of $\beta$-catenin overexpression or mutations. All eight patients with $\beta$-catenin mutations were alive and well after a median follow-up of 6 years. These data confirm the previous hypothesis that $\beta$-catenin mutations may represent a different pathway in endometrial carcinogenesis with a distinct morphologic appearance (squamous differentiation) and good prognosis, including low-grade and stage tumors at presentation. $^{44}$
Several groups have tried to determine the molecular characteristics of tamoxifen-associated endometrial carcinoma with inconclusive results. Some authors have noted that these tumors were particularly associated with $K$-ras mutations. ${ }^{45-47}$ The relationship between estrogen and $K$-ras is not well defined. In vitro experiments have shown that enhanced transcriptional activation of estrogen receptor- $\alpha$ contributed to a $K$-ras-mediated cell line transformation, while inactivation of estrogen receptor- $\alpha$ in the presence of mutant K-ras leads to senescence and death of a cell line. ${ }^{48,49}$ In vivo, a study of endometrial polyps in patients treated with tamoxifen showed that the frequency of $K$-ras mutations was $64 \%$, a significantly higher value than in sporadic endometrial hyperplasia. ${ }^{46}$ Others have found that $43 \%$ of women treated with tamoxifen or a derivative drug toremifene developed a de novo K-ras mutation in a seemingly normal endometrial tissue ${ }^{47}$ However, in a study from the database of Memorial Sloan-Kettering Cancer Center, the distribution of mutations in K-Ras was very similar in patients with history of tamoxifen exposure and patients with sporadic endometrial carcinoma. ${ }^{50}$ We encountered a possible association between $K$-ras mutations and exposure to tamoxifen (found in $21 \%$ of patients with history of tamoxifen exposure $v s 7 \%$ of patients in the control groups). In our study, the rate of $K$-ras mutations in the ovarian hyperthecosis/adult granulosa cell tumor group was almost identical to the one in the random control group ( $6 \mathrm{vs} 7 \%$ ) arguing against a significant effect of endogenous estrogen on the rate of $K$-ras mutations. No statistical significance was achieved for these findings, probably due to our limited sample. However, if these results were to be confirmed in a larger study, it could suggest that K-ras mutations in tamoxifen-induced endometrial carcinoma are not due to its estrogenic action (as seems to be the case with $\beta$-catenin mutations), but rather to a particular, tamoxifen-specific pathway, independent of estrogen.

The study from Memorial Sloan-Kettering Cancer Center has also found no difference in the rate of mutations in PTEN and presence of MSI between patients with history of tamoxifen exposure and patients with sporadic endometrial carcinoma. ${ }^{50}$ Another group also obtained similar results for expression of PTEN. ${ }^{51}$ According to the literature, PTEN expression varies through the menstrual cycle and early pregnancy; it has been noted to be elevated by estrogens and reduced by progestins during physiological hormonal fluctuations. ${ }^{19}$ In endometrial carcinoma, PTEN mutations are frequently seen in early stage disease and even in morphologically normal endometrium predating tumor development; in addition, it commonly coexists with endometrial hyperplasia, suggesting that PTEN mutations are an early event in the development of endometrioid endometrial carcinoma. ${ }^{5,16,52}$ It has been shown in vitro that estradiol may downregulate PTEN activity 
by increasing its phosphorylation in normal human endometrial cells. ${ }^{17}$ It has also been proposed that estradiol may directly affect PI3K-related signaling pathway by increasing the phosphorylation of Akt in endometrial cells. ${ }^{18}$ Loss of PTEN in mouse endometrium has lead to increased phosphorylation of estrogen receptor- $\alpha$, which resulted in activation of this nuclear receptor even in the absence of ligand, leading to enhanced ability to activate the transcription of its target genes, and therefore contribute to the neoplastic transformation of the endometrial cells. ${ }^{53}$ Even though PTEN mutations are frequently present in tumors with MSI, little is known about the interrelation between MSI and estrogen exposure. MSI positive colon cancers have been associated with very low levels of estrogen receptors ${ }^{54}$ while studies in breast carcinoma have yielded controversial results. ${ }^{55,56}$ However, to the best of our knowledge, there is no data analyzing the relationship between MSI and estrogen exposure in endometrial carcinoma. In our study, it appears that patients with tamoxifen exposure had a lower prevalence of PTEN mutations (9 vs $40 \%$ in the control group) and MSI (8 vs 29\% among controls). As was the case with $K$-ras, the difference was not statistically significant, but it suggests that tamoxifen may be carcinogenic to endometrial cells through a different mechanism from estrogen and may have a lower potential for causing PTEN and MSI-associated EC.

Finally, cases of carcinosarcoma, clear cell carcinoma and mixed carcinoma were included in the study, as these tumors are rare and their molecular profile is not well known. A mixed carcinoma showed a Gly(GGT)12(GAT)Asp K-ras mutation, identical to one seen in two other cases of pure endometrioid endometrial carcinoma. Clear cell carcinoma and carcinosarcoma had no detectable abnormalities. The significance of these findings is unknown and more studies specifically focusing on these lesions are in order.

Our study has several limitations. The size of the cohort was not large enough to obtain statistical significance on several values, such as the expression of $K$-ras and PTEN mutations and MSI in patients with history of tamoxifen exposure. Selecting the control groups also posed difficulties as this was a retrospective study based on archival material and very limited information on clinical history was available. We searched to compensate for this deficit by carefully choosing patients with pathologic evidence of ovarian hyperthecosis and adult granulosa cell tumor, as a 'hyperestrogenic control' and a random group of patients without pathologic or clinical evidence of increased estrogen exposure as a 'random control'. However, serum estrogen levels were not assayed in any of the patients; therefore, we can not be absolutely certain that all patients in the random group had normal levels of estrogens and alternatively that all patients in the ovarian hyperthecosis/adult granulosa cell tumor group had significantly increased serum levels of estrogens.

In conclusion, the results of our study suggest that there is a strong and significant direct relationship between history of tamoxifen exposure and overexpression of $\beta$-catenin in endometrial carcinoma. The prevalence of $\beta$-catenin mutations was also high in patients with ovarian hyperthecosis and adult granulosa cell tumor as opposed to random controls, suggesting it was due to direct estrogen receptormediated carcinogenic effect of tamoxifen and estrogen on endometrial cells and not to DNA damage by tamoxifen. Our findings are in keeping with previous research indicating that mutations in $\beta$-catenin could represent a separate pathway in endometrial carcinogenesis resulting in low-grade, low-stage endometrioid endometrial carcinoma with squamous differentiation and a good prognosis. On the other hand, there was a trend for increased rate of $K$-ras mutations as well as decreased frequency of PTEN mutations and MSI in patients with tamoxifen exposure although additional studies with a larger group of patients are needed to confirm these findings.

\section{Disclosure/Conflicts of interest}

This work was partially supported by research Grants RETICS RD06/0020/0015, Department of Health, Spain and Fundació La Marató de TV3Proyecto: 050432. GM is a junior investigator of the 'Ramón y Cajal Program' of the Spanish Ministry of Education and Science (2004).

\section{References}

1 Bokhman JV. Two pathogenetic types of endometrial carcinoma. Gynecol Oncol 1983;15:10-17.

2 Lax SF. Molecular genetic pathways in various types of endometrial carcinoma: from a phenotypical to a molecular-based classification. Virchows Arch 2004;444:213-223.

3 Matias-Guiu X, Catasus L, Bussaglia E, et al. Molecular pathology of endometrial hyperplasia and carcinoma. Hum Pathol 2001;32:569-577.

4 Bilbao C, Rodriguez G, Ramirez R, et al. The relationship between microsatellite instability and PTEN gene mutations in endometrial cancer. Int J Cancer 2006;119:563-570.

5 Mutter GL, Lin MC, Fitzgerald JT, et al. Altered PTEN expression as a diagnostic marker for the earliest endometrial precancers. J Natl Cancer Inst 2000;92:924-930.

6 Fukuchi T, Sakamoto M, Tsuda H, et al. Beta-catenin mutation in carcinoma of the uterine endometrium. Cancer Res 1998;58:3526-3528.

7 Lax SF, Kendall B, Tashiro H, et al. The frequency of p53, K-ras mutations, and microsatellite instability differs in uterine endometrioid and serous carcinoma: evidence of distinct molecular genetic pathways. Cancer 2000;88:814-824. 
8 Palacios J, Catasus L, Moreno-Bueno G, et al. Beta- and gamma-catenin expression in endometrial carcinoma. Relationship with clinicopathological features and microsatellite instability. Virchows Arch 2001;438: 464-469.

9 Saegusa M, Hashimura M, Yoshida T, et al. Betacatenin mutations and aberrant nuclear expression during endometrial tumorigenesis. Br J Cancer 2001;84 :209-217.

10 Caduff RF, Johnston CM, Frank TS. Mutations of the K-ras oncogene in carcinoma of the endometrium. Am J Pathol 1995;146:182-188.

11 Enomoto $\mathrm{T}$, Inoue $\mathrm{M}$, Perantoni $\mathrm{AO}$, et al. K-ras activation in neoplasms of the human female reproductive tract. Cancer Res 1990;50:6139-6145.

12 Sasaki H, Nishii H, Takahashi H, et al. Mutation of the K-ras protooncogene in human endometrial hyperplasia and carcinoma. Cancer Res 1993;53:1906-1910.

13 Black D, Soslow RA, Levine DA, et al. Clinicopathologic significance of defective DNA mismatch repair in endometrial carcinoma. J Clin Oncol 2006;24: 1745-1753.

14 Henderson BE, Feigelson HS. Hormonal carcinogenesis. Carcinogenesis 2000;21:427-433.

15 Key TJ, Pike MC. The dose-effect relationship between 'unopposed' oestrogens and endometrial mitotic rate: its central role in explaining and predicting endometrial cancer risk. Br J Cancer 1988;57:205-212.

16 Hecht JL, Mutter GL. Molecular and pathologic aspects of endometrial carcinogenesis. J Clin Oncol 2006;24: 4783-4791.

17 Guzeloglu-Kayisli O, Kayisli UA, Al-Rejjal R, et al. Regulation of PTEN (phosphatase and tensin homolog deleted on chromosome 10) expression by estradiol and progesterone in human endometrium. J Clin Endocrinol Metab 2003;88:5017-5026.

18 Guzeloglu Kayisli O, Kayisli UA, Luleci G, et al. In vivo and in vitro regulation of Akt activation in human endometrial cells is estrogen dependent. Biol Reprod 2004;71:714-721.

19 Mutter GL, Lin MC, Fitzgerald JT, et al. Changes in endometrial PTEN expression throughout the human menstrual cycle. J Clin Endocrinol Metab 2000;85: 2334-2338.

20 Goldstein SR. The effect of SERMs on the endometrium. Ann N Y Acad Sci 2001;949:237-242.

21 ACOG committee opinion. No. 336: Tamoxifen and uterine cancer. Obstet Gynecol 2006;107:1475-1478.

22 Deligdisch L, Kalir T, Cohen CJ, et al. Endometrial histopathology in 700 patients treated with tamoxifen for breast cancer. Gynecol Oncol 2000;78:181-186.

23 Fisher B, Costantino JP, Wickerham DL, et al. Tamoxifen for prevention of breast cancer: report of the National Surgical Adjuvant Breast and Bowel Project P-1 Study. J Natl Cancer Inst 1998;90: 1371-1388.

24 Mourits MJ, Ten Hoor KA, van der Zee AG, et al. The effects of tamoxifen on proliferation and steroid receptor expression in postmenopausal endometrium. J Clin Pathol 2002;55:514-519.

25 Schwartz LB, Krey L, Demopoulos R, et al. Alterations in steroid hormone receptors in the tamoxifen-treated endometrium. Am J Obstet Gynecol 1997;176: 129-137.

$26 \mathrm{Wu}$ H, Chen Y, Liang J, et al. Hypomethylation-linked activation of PAX2 mediates tamoxifen-stimulated endometrial carcinogenesis. Nature 2005;438:981-987.
27 Shang Y. Molecular mechanisms of oestrogen and SERMs in endometrial carcinogenesis. Nature Rev 2006;6:360-368.

28 Shang Y. Hormones and cancer. Cell Research 2007; $17: 277-279$

29 Bartsch H, Phillips DH, Nair J, et al. Lack of evidence for tamoxifen- and toremifene-DNA adducts in lymphocytes of treated patients. Carcinogenesis 2000; 21:845-847.

30 Carmichael PL, Sardar S, Crooks N, et al. Lack of evidence from HPLC 32P-post-labelling for tamoxifenDNA adducts in the human endometrium. Carcinogenesis 1999;20:339-342.

31 Beland FA, McDaniel LP, Marques MM. Comparison of the DNA adducts formed by tamoxifen and 4-hydroxytamoxifen in vivo. Carcinogenesis 1999;20:471-477.

32 Schild LJ, Divi RL, Beland FA, et al. Formation of tamoxifen-DNA adducts in multiple organs of adult female cynomolgus monkeys dosed with tamoxifen for 30 days. Cancer Res 2003;63:5999-6003.

33 Shibutani S, Suzuki N, Laxmi YR, et al. Identification of tamoxifen-DNA adducts in monkeys treated with tamoxifen. Cancer Res 2003;63:4402-4406.

34 Tavasolli F, Devilee P. WHO Classification of Tumours. Pathology and Genetics of Tumours of the Breast and Female Genital Organs. IARC Press: Lyon, 2003, pp 221-228.

35 Moreno-Bueno G, Hardisson D, Sanchez C, et al. Abnormalities of the APC/beta-catenin pathway in endometrial cancer. Oncogene 2002;21:7981-7990.

36 Dahia PL, Marsh DJ, Zheng Z, et al. Somatic deletions and mutations in the Cowden disease gene, PTEN, in sporadic thyroid tumors. Cancer Res 1997;57: $4710-4713$.

37 Shibutani S, Ravindernath A, Suzuki N, et al. Identification of tamoxifen-DNA adducts in the endometrium of women treated with tamoxifen. Carcinogenesis 2000;21:1461-1467.

38 Terashima I, Suzuki N, Shibutani S. Mutagenic potential of alpha-(N2-deoxyguanosinyl) tamoxifen lesions, the major DNA adducts detected in endometrial tissues of patients treated with tamoxifen. Cancer Res 1999;59:2091-2095.

39 Hou X, Tan Y, Li M, et al. Canonical Wnt signaling is critical to estrogen-mediated uterine growth. Mol Endocrin 2004;18:3035-3049.

40 Katoh M. Expression and regulation of WNT1 in human cancer: up-regulation of WNT1 by betaestradiol in MCF-7 cells. Int J Oncol 2003;22:209-212.

41 Easwaran V, Pishvaian M, Salimuddin, et al. Crossregulation of beta-catenin-LEF/TCF and retinoid signaling pathways. Curr Biol 1999;9:1415-1418.

42 Truica CI, Byers S, Gelmann EP. Beta-catenin affects androgen receptor transcriptional activity and ligand specificity. Cancer Res 2000;60:4709-4713.

43 Gielen SC, Kuhne LC, Ewing PC, et al. Tamoxifen treatment for breast cancer enforces a distinct geneexpression profile on the human endometrium: an exploratory study. Endocr Relat Cancer 2005;12: 1037-1049.

44 Brachtel EF, Sanchez-Estevez C, Moreno-Bueno G, et al. Distinct molecular alterations in complex endometrial hyperplasia $(\mathrm{CEH})$ with and without immature squamous metaplasia (squamous morules). Am J Surg Path 2005;29:1322-1329.

45 Esteller M, Garcia A, Martinez-Palones JM, et al. Endometrial carcinoma in tamoxifen-treated breast 
cancer patient: clinicopathological, immunohistochemical, and genetic analysis. Int J Gynecol Pathol 1999;18:387-391.

46 Hachisuga T, Miyakawa T, Tsujioka H, et al. K-ras mutation in tamoxifen-related endometrial polyps. Cancer 2003;98:1890-1897.

47 Wallen M, Tomas E, Visakorpi T, et al. Endometrial K-ras mutations in postmenopausal breast cancer patients treated with adjuvant tamoxifen or toremifene. Cancer Chemother Pharmacol 2005;55: 343-346.

48 Kato K, Horiuchi S, Takahashi A, et al. Contribution of estrogen receptor alpha to oncogenic K-Ras-mediated NIH3T3 cell transformation and its implication for escape from senescence by modulating the p53 pathway. J Biol Chem 2002;277:11217-11224.

49 Kato K, Ueoka Y, Kato K, et al. Contribution of enhanced transcriptional activation by ER to [12Val] K-Ras mediated NIH3T3 cell transformation. Oncogene 1997;15:3037-3046.

50 Prasad M, Wang H, Douglas W, et al. Molecular genetic characterization of tamoxifen-associated endometrial cancer. Gynecol Oncol 2005;96:25-31.
51 Holtz D, Ramondetta LM, Burke TW, et al. PTEN expression in tamoxifen-associated endometrial cancers. Anticancer Res 2002;22:2945-2948.

52 Mutter GL, Ince TA, Baak JP, et al. Molecular identification of latent precancers in histologically normal endometrium. Cancer Res 2001;61:4311-4314.

53 Vilgelm A, Lian Z, Wang H, et al. Akt-mediated phosphorylation and activation of estrogen receptor alpha is required for endometrial neoplastic transformation in Pten+/- mice. Cancer Res 2006;66:3375-3380.

54 Notarnicola M, Gristina R, Messa C, et al. Oestrogen receptors and microsatellite instability in colorectal carcinoma patients. Cancer Lett 2001;168:65-70.

55 Caldes T, Perez-Segura P, Tosar A, et al. Microsatellite instability correlates with negative expression of estrogen and progesterone receptors in sporadic breast cancer. Teratog Carcinog Mutagen 2000;20:283-291.

56 Shen KL, Yang LS, Hsieh HF, et al. Microsatellite alterations on human chromosome 11 in in situ and invasive breast cancer: a microdissection microsatellite analysis and correlation with p53, ER (estrogen receptor), and PR (progesterone receptor) protein immunoreactivity. J Surg Oncol 2000;74:100-107. 